\title{
Minimally invasive esophagectomy for esophageal cancer in the People's Republic of China: an overview
}

This article was published in the following Dove Press journal:

OncoTargets and Therapy

I March 2013

Number of times this article has been viewed

\author{
Chengchu Zhu' \\ Ketao Jin ${ }^{2}$ \\ 'Department of Cardiothoracic \\ Surgery, ${ }^{2}$ Department of Surgical \\ Oncology, Taizhou Hospital, Wenzhou \\ Medical College, Linhai, Zhejiang, \\ People's Republic of China
}

\begin{abstract}
Since its introduction in the People's Republic of China in 1992, minimally invasive esophagectomy (MIE) has shown the classical advantages of minimally invasive surgery over its open counterpart. Like all pioneers of the technique, cardiothoracic surgeons in the People's Republic of China claim that MIE has a lower risk of pulmonary infection, faster recovery, a shorter hospital stay, and a more rapid return to daily activities than open esophagectomy, while offering the same functional and oncologic results. There has been burgeoning interest in MIE in the People's Republic of China since 1995. The last decade has witnessed nationwide growth in the application of MIE and yielded a significant amount of scientific data in support of its clinical merits and advantages. However, no prospective randomized controlled trials have actually investigated the benefits of MIE in the People's Republic of China. Here we review the current data and state of the art MIE treatment for esophageal cancer in the People's Republic of China.
\end{abstract}

Keywords: esophagectomy, minimally invasive esophagectomy, esophageal cancer, review

\section{Introduction}

The global incidence of esophageal cancer has increased by $50 \%$ in the past two decades. ${ }^{1,2}$ Advances in neoadjuvant and adjuvant chemotherapy and chemoradiotherapy have led to increasingly multimodal treatment for patients with esophageal cancer, which has decreased the rate of local recurrence and improved long-term survival for some patients. However, surgical resection with radical lymphadenectomy is regarded as one of the curative options for resectable esophageal cancer. ${ }^{3-6}$ Frequently, due consideration of surgical resection may not be given because of concerns with regard to the morbidity of open esophagectomy.

In an effort to decrease the morbidity associated with open esophagectomy, Chinese surgeons have adopted a minimally invasive approach to esophageal resection. Because of the potential advantages, including avoiding thoracotomy and laparotomy and reducing the rate of pulmonary infections (thus reducing the inpatient stay), ${ }^{7,8}$ minimally invasive esophagectomy (MIE) was introduced into clinical practice in Taiwan ${ }^{9}$ in 1992 at the same time as in Western countries, ${ }^{10-12}$ was gradually implemented, and is now a commonplace procedure in the People's Republic of China (Figure 1), including in Beijing, ${ }^{13-15}$ Jinan in Shandong Province, ${ }^{16}$ Zhengzhou in Henan Province, ${ }^{17}$ Nanjing in Jiangsu Province, ${ }^{18}$ Shanghai, ${ }^{19-30}$ Taizhou in Zhejiang Province, ${ }^{31-33}$ Fuzhou in Fujian Province, ${ }^{34-36}$ Taipei $^{9}$ and Taichung ${ }^{37}$ in Taiwan, Hongkong, ${ }^{38-40}$ Guangzhou in Guangdong Province, ${ }^{41,42}$ Changsha in Hunan Province, ${ }^{43}$ Chongqing, ${ }^{44,45}$ and Chengdu ${ }^{46-48}$ and Nanchong ${ }^{49}$ in Sichuan Province. There has been burgeoning interest in MIE since
Correspondence: Ketao jin

Department of Surgical Oncology,

Taizhou Hospital, Wenzhou Medical

College, I 50 Ximen Road, Linhai,

Zhejiang 317000, People's Republic

of China

Tel +8657685199876

Fax +8657685199876

Email jinketao200।@zju.edu.cn 


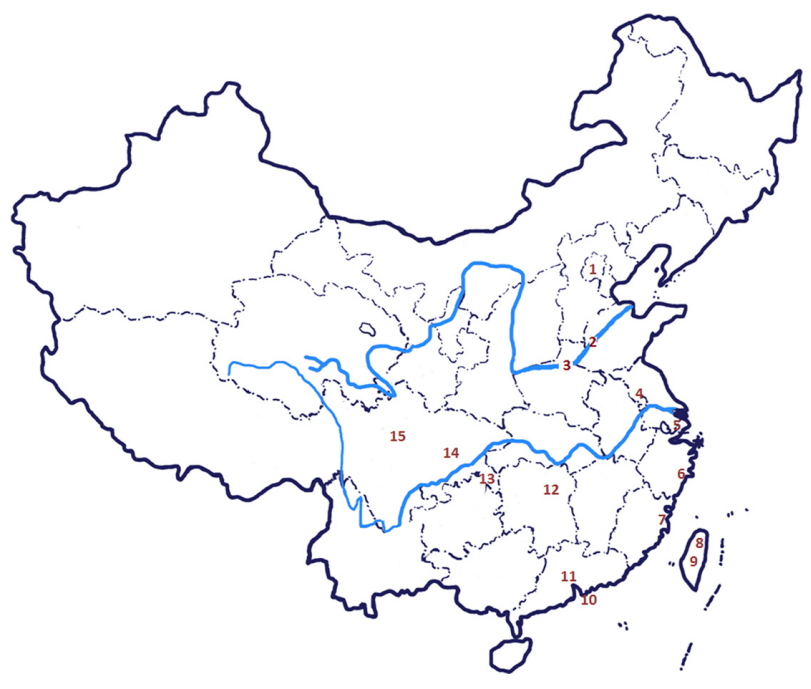

Figure I Fifteen national areas implementing minimally invasive esophagectomy in the People's Republic of China.

Notes: High-volume centers: First Affiliated Hospital, School of Medicine, Peking University and Chaoyang Hospital, Capital Medical University in Beijing; Shandong Provincial Hospital, Shandong University in Jinan; The Affiliated Tumor Hospital, Zhengzhou University in Zhengzhou; Jiangsu Provincial Cancer Hospital in Nanjing; Zhongshan Hospital, Fudan University, The Cancer Hospital of Fudan University, Changzheng Hospital, Second Military Medical University and Shanghai Chest Hospital, Shanghai Jiaotong University in Shanghai; Taizhou Hospital, Wenzhou Medical College in Taizhou; Affiliated Union Hospital of Fujian Medical University and Fujian Provincial Tumor Hospital of Fujian Medical University in Fuzhou; Chang Gung Memorial Hospital, Chang Gung Medical College in Taipei; Tungs' Taichung MetroHarbor Hospital in Taichung; University of Hong Kong Medical Centre, Queen Mary Hospital and The Chinese University of Hong Kong, Prince of Wales Hospital in Hongkong; Nanfang Hospital, Southern Medical University and Cancer Center, Sun Yatsen University in Guangzhou; Second Xiangya Hospital of Central South University in Changsha; Daping Hospital, Third Military Medical University in Chongqing; West China Hospital, Sichuan University in Chengdu; The Second Clinical Institute, North Sichuan Medical College in Nanchong.

it was first described in Taiwan in $1995^{\circ}$ and in the People's Republic of China in 1999. ${ }^{13}$ The last decade has witnessed nationwide growth in use of MIE, yielding a significant amount of scientific data to support its clinical merits and advantages. Here we review the current data and state of the art for MIE in the treatment of esophageal cancer in the People's Republic of China.

\section{Literature on MIE in the People's Republic of China}

The current literature was reviewed by searching the PubMed/ Medline database from January 1992 to December 2012 using keywords such as "minimally invasive oesophagectomy", "MIE", and "China". Sixty-one full articles were found to be relevant to MIE (Figure 2). A total of 33 publications (54.1\%) were in English. However, nearly half of all relevant clinical reports $(28,45.9 \%)$ were published in Chinese, despite the fact that it has been necessary to report the current status of MIE as performed in the People's Republic of China to cardiotho-

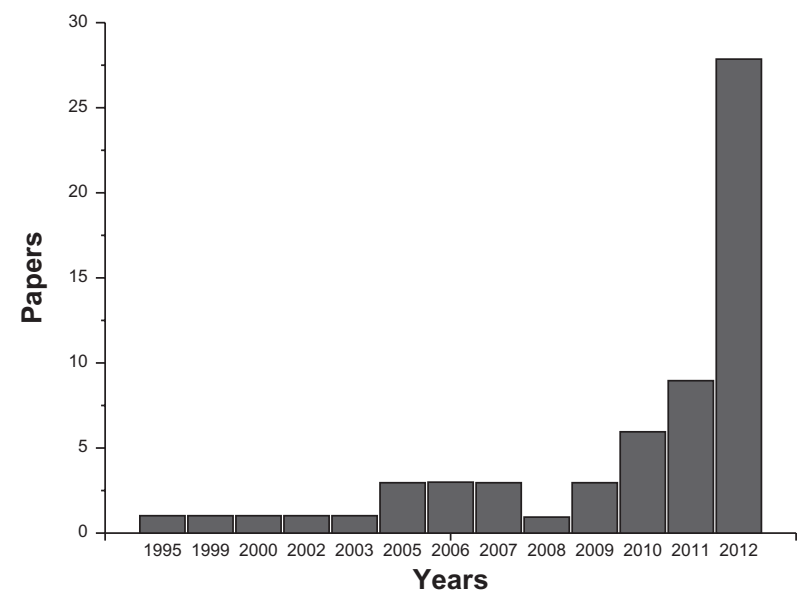

Figure 2 Numbers of papers related to minimally invasive esophagectomy performed in the People's Republic of China identified in the PubMed/Medline database, using keywords such as "minimally invasive oesophagectomy", "MIE", and "China".

racic surgeons worldwide. A marked increase in the number of papers dedicated to MIE was observed from 2010 to 2012 (Figure 2), which probably reflects increased research interest among the surgical community and wider clinical application of this patient-friendly approach.

\section{Operative data on MIE}

Key outcomes of the major studies are summarized in the Tables 1 and 2. Thirty-two relevant papers, consisting of prospective and retrospective studies, were identified. Eight papers directly compared open oesophagectomy and MIE, and ${ }^{16,17,21,30,31,39,41,48}$ five of these involved studies performed prospectively. ${ }^{16,31,39,41,48}$ Common outcome measures included operative data (operative time, blood loss, conversion rate), morbidity (duration of intensive care and total hospital stay), complications (pulmonary complications, anastomotic leaks, chylothorax), mortality data, and follow-up periods. Neoadjuvant treatment numbers were included for each study.

\section{Surgical approaches}

Surgical approaches for MIE performed by Chinese cardiothoracic surgeons are multiple and complicated. As listed in Table 1, the majority of centers use mainly total MIE (laparoscopic and thoracoscopic esophagectomy), whereas hybrid MIE (thoracoscopy and laparotomy/laparoscopy and thoracotomy) is used in routine practice in some centers. At our center, we originally used hybrid $\mathrm{MIE}^{31}$ but more recently transitioned to a minimally invasive modified McKeown 3-incision total MIE (laparoscopic and thoracoscopic esophagectomy) in $2010 .^{32,33}$ 
Table I Survey over major reports of minimally invasive esophagectomy in the People's Republic of China: operative data

\begin{tabular}{|c|c|c|c|c|c|c|c|c|c|}
\hline Reference & PS/RS & Patient (n) & AC & TC & AS & Position & От (min) & BL (mL) & C, n (\%) \\
\hline Liu et al ${ }^{9}$ & RS & 20 & Open & MI & Thoracic & Left Lateral & 280 & 250 & NA \\
\hline Li et $\mathrm{al}^{14}$ & PS & 6 & MI & MI & Thoracic & Left Lateral & $260 \pm 42$ & $520 \pm 160$ & 0 \\
\hline Li et al ${ }^{15}$ & RS & 6 & MI & MI & Thoracic & Left Lateral & 380 & 300 & 0 \\
\hline \multirow[t]{2}{*}{ Du et al ${ }^{16}$} & PS & 45 & Open & $\mathrm{HA}$ & $\begin{array}{l}\text { Cervical/ } \\
\text { thoracic }\end{array}$ & Left Lateral & $29 \pm 5(\mathrm{TC})$ & $93 \pm 19(\mathrm{TC})$ & NA \\
\hline & & 27 & Open & MI & Cervical & Left Lateral & $425(240-538)$ & $400(100-1200)$ & I (4) \\
\hline Liu et $\mathrm{al}^{17}$ & RS & 98 & MI & MI & Cervical & Left Lateral & $134.5 \pm 42.3$ & $85.1 \pm 32.8$ & NA \\
\hline \multirow[t]{2}{*}{ Hou et $\mathrm{al}^{18}$} & RS & 41 & MI & MI & Cervical & Prone & $230(170-310)$ & $275(100-320)$ & NA \\
\hline & & 41 & MI & MI & Cervical & Left Lateral & $280(190-380)$ & $360(120-670)$ & NA \\
\hline \multirow[t]{2}{*}{ Wu et a ${ }^{20}$} & PS & 32 & Open & MI & Cervical & Supine & 180 & 218 & 0 \\
\hline & & 8 & MI & MI & Cervical & Supine & 220 & 100 & 0 \\
\hline Zhou et $\mathrm{a}^{22}$ & PS & 30 & Open & MI & Cervical & Left Lateral & $225(195-290)$ & $250 \pm 52.2$ & I (3) \\
\hline Tan et $\mathrm{al}^{23}$ & RS & 36 & Open & MI & Cervical & Left Lateral & $250(190-330)$ & $165(100-350)$ & 0 \\
\hline Wang et $\mathrm{a}^{24}$ & PS & 27 & MI & MI & Cervical & Left Lateral & $267 \pm 5 I$ & $327 \pm 83$ & NA \\
\hline \multirow[t]{2}{*}{ Feng et $\mathrm{a}^{25}$} & PS & 27 & MI & MI & Cervical & Supine & $194.4 \pm 26$ & $215 \pm 111.6$ & 0 \\
\hline & & 27 & MI & MI & Cervical & Left Lateral & $228.1 \pm 35.8$ & $142.6 \pm 51.3$ & 0 \\
\hline \multirow[t]{2}{*}{ Wang et $\mathrm{a}^{26}$} & RS & $48^{\mathrm{a}}$ & MI & MI & Cervical & Left Lateral & $279 \pm 64$ & $359 \pm 156$ & NA \\
\hline & & $49^{b}$ & MI & MI & Cervical & Left Lateral & $266 \pm 56$ & $336 \pm 130$ & NA \\
\hline \multirow[t]{2}{*}{ Feng et $\mathrm{al}^{27}$} & RS & 52 & MI & MI & Cervical & Left Lateral & $82 \pm 17(\mathrm{TC})$ & $139 \pm 54(\mathrm{TC})$ & 0 \\
\hline & RS & 36 & MI & MI & Cervical & Prone & $70 \pm 20(\mathrm{TC})$ & $100 \pm 52(\mathrm{TC})$ & 0 \\
\hline Shen et $\mathrm{a}^{28}$ & RS & 76 & MI & MI & Cervical & Prone & $89 \pm 32(\mathrm{TC})$ & $152 \pm 108$ & 0 \\
\hline \multirow[t]{2}{*}{ Feng et $\mathrm{al}^{29}$} & PS & 41 & MI & MI & Cervical & Decubitus & $217 \pm 32$ & $142 \pm 49$ & I (2.4) \\
\hline & PS & 52 & MI & MI & Cervical & Prone & $202 \pm 21$ & $123 \pm 56$ & 0 \\
\hline Wang et $\mathrm{a}^{30}$ & RS & 260 & $\mathrm{MI} / \mathrm{O}$ & MI & Cervical & Left Lateral & $105 \pm 30(\mathrm{TC})$ & $95 \pm 48(\mathrm{TC})$ & NA \\
\hline Zhu et $\mathrm{al}^{31}$ & PS & 25 & Open & MI & Thoracic & Left Lateral & $88 \pm 15(\mathrm{TC})$ & $280 \pm 132(\mathrm{TC})$ & NA \\
\hline Chen et $\mathrm{a}^{32}$ & PS & 67 & MI & MI & Cervical & Left Lateral & $274 \pm 15$ & $225 \pm 31$ & NA \\
\hline Zhu et $\mathrm{al}^{33}$ & PS & 11 & MI & MI & Cervical & Left Lateral & $242.3 \pm 27.0$ & $168.2 \pm 95.6$ & NA \\
\hline Lin et $\mathrm{al}^{34}$ & RS & 80 & MI & MI & Cervical & Left Lateral & NA & $100-250$ & $6(8)$ \\
\hline Liu et $\mathrm{al}^{35}$ & RS & 297 & MI & MI & Cervical & Left Lateral & $242.3 \pm 58.7$ & NA & I (3) \\
\hline Lin et $\mathrm{al}^{36}$ & RS & 150 & MI & MI & Cervical & Left Lateral & $258 \pm 45$ & $207 \pm 130$ & $6(4)$ \\
\hline Cense et $\mathrm{a}^{38}$ & PS & 30 & Open & MI & Cervical & Left Lateral & $400(180-570)$ & $700(164-3000)$ & $2(7)$ \\
\hline Law et $\mathrm{a}^{39}$ & PS & 30 & Open & MI & Thoracic & $\begin{array}{l}\text { Left Lateral (29)/ } \\
\text { prone (I) }\end{array}$ & $392(180-570)$ & $700(164-3000)$ & $2(6.7)$ \\
\hline Wong et $\mathrm{al}^{40}$ & PS & 12 & MI & MI & Thoracic & Supine & $510(300-660)$ & $500(250-2500)$ & I (8) \\
\hline Wang et $\mathrm{a}^{41}$ & PS & 33 & MI & MI & Cervical & $\mathrm{Na}$ & NA & NA & NA \\
\hline Xie et al ${ }^{42}$ & RS & 100 & MI & MI & Cervical & Left Lateral & 310 & 200 & $4(4)$ \\
\hline \multirow[t]{2}{*}{ Yuan et $\mathrm{al}^{43}$} & PS & 32 & MI & MI & Cervical & Left Lateral & $290.8 \pm 36.9$ & NA & NA \\
\hline & & 36 & Open & MI & Cervical & Supine & $249.0 \pm 31.0$ & NA & NA \\
\hline Guo et $\mathrm{al}^{44}$ & RS & 89 & Open & MI & Cervical & Left Lateral & $323.7 \pm 50.3$ & $307.8 \pm 162.7$ & $8(9.0)$ \\
\hline Guo et $\mathrm{al}^{45}$ & RS & 135 & Open & MI & Cervical & Left Lateral & $334 \pm 51.1$ & $349.3 \pm 164.8$ & $10(7.4)$ \\
\hline Zhang et $\mathrm{al}^{46}$ & RS & 160 & MI & MI & Cervical & Prone & $230-780$ & $20-4000$ & $9(5.6)$ \\
\hline Gao et al ${ }^{48}$ & PS & 96 & MI & MI & Cervical & Left Lateral & $330.2 \pm 36.7$ & $346.7 \pm 41.1$ & 0 \\
\hline
\end{tabular}

Notes: aRetrosternal route of gastric tube reconstruction; ${ }^{b}$ prevertebral route of gastric tube reconstruction.

Abbreviations: PS, prospective study; RS, retrospective study; AC, abdominal component; TC, thoracic component; AS, anastomosis site; OT, operation time; BL, blood loss; C, conversion rate; MI, minimally invasive; $\mathrm{O}$, open; NA, not available; $\mathrm{HA}$, hand-assisted.

\section{Operative time and blood loss}

Operative time varied significantly between the studies, reflecting the type of MIE performed as well as accumulated experience and technical skills (Table 1). Blood loss also varied significantly from center to center, comprising around 100-700 mL (Table 1). Major blood loss and need for blood transfusion in particular increased the risk of postoperative morbidity and mortality.

\section{Conversion to open esophagectomy}

The conversion rate reported in the literature is in a range of $0 \%-9.7 \%$ (Table 1). However, with surgical experience, the conversion rate reduces and currently does not exceed 5\% in expert centers in the People's Republic of China. The main reason for conversion was bleeding. It is not appropriate to consider conversion from MIE to open esophagectomy as a failure because patient safety and the 
Table 2 Survey of major reports of minimally invasive esophagectomy in the People's Republic of China: mortality, morbidity, and postoperative complications

\begin{tabular}{|c|c|c|c|c|c|c|c|c|c|}
\hline Reference & Patient (n) & $\begin{array}{l}\text { AL, } \\
\text { n (\%) }\end{array}$ & $\begin{array}{l}\text { PC, } \\
\text { n (\%) }\end{array}$ & $\begin{array}{l}\text { Ch, } \\
\text { n (\%) }\end{array}$ & ICUS (d) & HS (d) & 30-DM & $\begin{array}{l}\text { Mortality, } \\
\text { n (\%) }\end{array}$ & $F P(m)$ \\
\hline Liu et $\mathrm{al}^{9}$ & 20 & 0 & 0 & 0 & NA & 19 & NA & NA & 11.5 \\
\hline Li et al ${ }^{14}$ & 6 & 0 & NA & NA & 0 & 17 & NA & NA & 2.5 \\
\hline Li et al ${ }^{15}$ & 6 & 0 & 0 & 0 & NA & NA & NA & NA & NA \\
\hline Du et $\mathrm{al}^{16}$ & 45 & NA & NA & NA & NA & $10.0 \pm 1.0$ & NA & NA & NA \\
\hline Liu et al ${ }^{17}$ & 98 & $2(2.0)$ & $10(10.2)$ & $3(3.1)$ & NA & $12.7 \pm 3.5$ & 1 & NA & NA \\
\hline \multirow[t]{2}{*}{ Hou et $\mathrm{al}^{18}$} & 41 & I (2.4) & $2(4.9)$ & 0 & NA & NA & NA & NA & 15.7 \\
\hline & 41 & I (2.4) & I (2.4) & $2(4.9)$ & NA & NA & NA & NA & 16.3 \\
\hline \multirow[t]{2}{*}{$W u$ et $a^{20}$} & 32 & $3(9.4)$ & I (3.I) & I (3.I) & 2.2 & 11.6 & NA & NA & NA \\
\hline & 8 & I (I2.5) & 0 & 0 & 1.2 & 10.6 & NA & NA & NA \\
\hline Zhou et a ${ }^{22}$ & 30 & $2(6.7)$ & $2(6.7)$ & I (3.3) & NA & $11.7 \pm 6.3$ & 0 & 0 & NA \\
\hline Tan et $\mathrm{a}^{23}$ & 36 & $5(13.9)$ & I (2.8) & I (2.8) & NA & 8.7 & 0 & 0 & NA \\
\hline Wang et $\mathrm{a}^{24}$ & 27 & $5(18.5)$ & I (3.7) & $2(7.4)$ & $2.3 \pm 1.7$ & NA & NA & NA & NA \\
\hline \multirow[t]{2}{*}{ Feng et $\mathrm{a}^{25}$} & 27 & $5(18.5)$ & 7 (25.9) & 0 & $3.1 \pm 4.4$ & $\mathrm{II} . \mathrm{I} \pm 6.6$ & 1 & NA & 36 \\
\hline & 27 & $4(14.8)$ & $4(14.8)$ & I (3.7) & $1.9 \pm 4.2$ & $13.3 \pm 10.6$ & 0 & NA & 36 \\
\hline \multirow[t]{2}{*}{ Wang et $\mathrm{a}^{26}$} & 48 & $10(20.8)$ & $2(4.2)$ & I (2.I) & $2.5 \pm 1.7$ & NA & 0 & NA & NA \\
\hline & 49 & $3(6.1)$ & $6(12.2)$ & I (2.0) & $2.8 \pm 1.9$ & NA & 0 & NA & NA \\
\hline \multirow[t]{2}{*}{ Feng et $\mathrm{al}^{27}$} & 52 & $8(I 5.4)$ & $5(9.6)$ & $2(3.8)$ & $1.3 \pm 3.5$ & $13.6 \pm 9.3$ & NA & NA & NA \\
\hline & 36 & $2(5.6)$ & I (2.8) & 0 & $\mathrm{I} .1 \pm 1.5$ & $10.9 \pm 6.0$ & NA & NA & NA \\
\hline Shen et $\mathrm{a}^{28}$ & 76 & $16(21.1)$ & $5(6.6)$ & I (I.3) & NA & $19.2 \pm 16.3$ & NA & 0 & NA \\
\hline \multirow[t]{2}{*}{ Feng et $\mathrm{al}^{29}$} & 41 & $9(22.0)$ & $4(9.8)$ & NA & $3.5 \pm 1.3$ & $17.4 \pm 12.5$ & NA & NA & NA \\
\hline & 52 & $4(7.7)$ & $5(9.6)$ & NA & $\mathrm{I} .5 \pm \mathrm{I} . \mathrm{I}$ & $11.4 \pm 6.8$ & NA & NA & NA \\
\hline Wang et a ${ }^{30}$ & 260 & $26(10)$ & $22(8.5)$ & $3(1.2)$ & NA & $\mid 4.3 \pm 7.5$ & NA & $2(7.7)$ & NA \\
\hline Zhu et a $\left.\right|^{31}$ & 25 & I (4) & NA & NA & NA & $10.9 \pm 2.5$ & NA & NA & NA \\
\hline Chen et a $\mathrm{a}^{32}$ & 67 & NA & $7(10.4)$ & NA & NA & $11.5 \pm 1.6$ & NA & NA & $14.0 \pm 2.2$ \\
\hline Zhu et $\mathrm{al}^{33}$ & 11 & $2(18.2)$ & $3(27.3)$ & NA & NA & $18.9 \pm 10.3$ & NA & NA & 4.5 \\
\hline Lin et $\mathrm{al}^{34}$ & 80 & I (I.3) & NA & $2(2.5)$ & NA & NA & NA & NA & NA \\
\hline Liu et $\mathrm{a}^{35}$ & 297 & $9(3.0)$ & $4 \mid(18.8)$ & NA & NA & $17.4 \pm 9.8$ & NA & NA & NA \\
\hline Lin et $\mathrm{al}^{36}$ & 150 & $9(6.0)$ & $17(11.3)$ & $5(3.3)$ & NA & NA & $2(1.3)$ & $9(6)$ & $3-22$ \\
\hline \multirow[t]{2}{*}{ Cense et a ${ }^{38}$} & 30 & I (3.3) & $12(40)$ & NA & NA & NA & NA & NA & NA \\
\hline & 27 & I (3.7) & $13(48.1)$ & NA & NA & NA & NA & $2(7)$ & NA \\
\hline Law et a $\left.\right|^{39}$ & 30 & I (3.3) & $12(40)$ & 0 & NA & NA & 1 & NA & NA \\
\hline Wong et $\mathrm{a}^{40}$ & 12 & I (8.3) & $2(17)$ & NA & 2 & 41 & 0 & 0 & NA \\
\hline Wang et a $\left.\right|^{41}$ & 33 & I (3.0) & 0 & 0 & NA & NA & NA & NA & NA \\
\hline Xie et $\mathrm{al}^{42}$ & 100 & II (II) & $13(13)$ & $3(3)$ & I (I) & $12(12)$ & NA & NA & NA \\
\hline \multirow[t]{2}{*}{ Yuan et $\mathrm{al}^{43}$} & 32 & $2(6.3)$ & NA & NA & 1 & $11.1 \pm 1.3$ & 0 & NA & NA \\
\hline & 36 & $5(\mid 3.9)$ & NA & NA & 1 & $11.6 \pm 1.7$ & 0 & NA & NA \\
\hline Guo et $\mathrm{al}^{44}$ & 89 & $6(6.7)$ & $4(4.5)$ & $4(4.5)$ & NA & $15.2 \pm 9.8$ & NA & NA & NA \\
\hline Guo et al ${ }^{45}$ & 135 & $9(6.7)$ & $7(5.2)$ & $8(5.9)$ & NA & NA & NA & NA & NA \\
\hline Zhang et $\mathrm{a}^{46}$ & 160 & $21(13.1)$ & $25(15.6)$ & $4(2.5)$ & 1 & 13.1 & $2(1.3)$ & $4(2.5)$ & NA \\
\hline Gao et al ${ }^{48}$ & 96 & $7(7.3)$ & $13(13.5)$ & I (I.I) & $19.2 \pm 3.5$ & $12.6 \pm 8.8$ & NA & $2(2.1)$ & NA \\
\hline
\end{tabular}

Abbreviations: AL, anastomotic leaks; PC, pulmonary complication; Ch, chylothorax; ICUS (d), intensive care unit stay (days); HS (d), hospital stay (days); 30-DM, 30-day mortality; FP (m), follow-up period (months); NA, not available.

oncologic integrity of the procedure should be of supreme importance.

\section{Mortality, morbidity, and postoperative complications}

Mortality rates following total MIE vary between $0 \%$ and $7.7 \%$ (Table 2), which compares favorably with an open transthoracic procedure mortality rate of $9.2 \%$ and an open transhiatal procedure mortality rate of $7.2 \%{ }^{50}$
However, at least half of the patients who undergo open oesophagectomy, performed through a right thoracotomy and laparotomy, are at risk of developing pulmonary complications requiring a protracted stay in intensive care, with consequences for quality of life during convalescence. ${ }^{50}$ Anastomotic leak is one of the most feared complications of MIE. From the operative data, the MIE leakage rate was in the range of $0 \%-20.8 \%$ (Table 2 ), which is comparable with the leakage rates reported for open oesophagectomy. ${ }^{51}$ 
Median duration of postoperative stay in intensive care following MIE was one day in the majority of studies (Table 2). MIE is associated with a significant reduction in hospital stay, with a mean postoperative stay of 12 days (Table 2).

\section{Outcomes}

There is little survival data for MIE available in the People's Republic of China. Only one study reported overall survival after MIE. ${ }^{25}$ Feng et al reported median survival for patients in a thoracoscope-assisted transthoracic esophagectomy group and in a mediastinoscope-assisted transhiatal esophagectomy group of 34.4 months and 36.8 months, respectively. ${ }^{25}$ There do not appear to be any prospective, randomized, controlled trials comparing the oncologic outcome of MIE with that of open esophagectomy. The present knowledge is based mainly on short-term, nonrandomized comparative studies or historical comparisons with outcomes of open surgery. ${ }^{31,39,41,48}$

\section{Conclusion}

In conclusion, MIE is becoming more popular in the People's Republic of China now that Chinese cardiothoracic surgeons are receiving adequate training in major centers. Use of the technique is growing in the People's Republic of China, as confirmed by the increasing number of recently published papers on MIE. However, no prospective, randomized, controlled trials have investigated the benefits of MIE in this country. Such trials, directly comparing MIE and open approaches, are urgently needed.

\section{Acknowledgments}

This work was supported by grants from Zhejiang Provincial Science and Technology Major Projects (2011C13039-2) and Zhejiang Provincial Science and Technology Innovation Team Projects (2011R09040-03).

\section{Disclosure}

The authors report no conflicts of interest in this work.

\section{References}

1. Jemal A, Bray F, Center MM, Ferlay J, Ward E, Forman D. Global cancer statistics. CA Cancer J Clin. 2011;61(2):69-90.

2. Parkin DM, Pisani P, Ferlay J. Global cancer statistics. $C A$ Cancer $J$ Clin. 1999;49(1):33-64.

3. Enzinger PC, Mayer RJ. Esophageal cancer. $N$ Engl J Med. 2003;349(23):2241-2252.

4. Sjoquist KM, Burmeister BH, Smithers BM, et al. Survival after neoadjuvant chemotherapy or chemoradiotherapy for resectable oesophageal carcinoma: an updated meta-analysis. Lancet Oncol. 2011;12(7): 681-692.
5. Cerfolio RJ, Bryant AS, Hawn MT. Technical aspects and early results of robotic esophagectomy with chest anastomosis. JThorac Cardiovasc Surg. 2013;145(1):90-96.

6. Kastenmeier A, Gonzales H, Gould JC. Robotic applications in the treatment of diseases of the esophagus. Surg Laparosc Endosc Percutan Tech. 2012;22(4):304-309.

7. Palanivelu C, Prakash A, Senthilkumar R, et al. Minimally invasive esophagectomy: thoracoscopic mobilization of the esophagus and mediastinal lymphadenectomy in prone position - experience of 130 patients. J Am Coll Surg. 2006;203(1):7-16.

8. Luketich JD, Alvelo-Rivera M, Buenaventura PO, et al. Minimally invasive esophagectomy: outcomes in 222 patients. Ann Surg. 2003;238(4): 486-494.

9. Liu HP, Chang CH, Lin PJ, Chang JP. Video-assisted endoscopic esophagectomy with stapled intrathoracic esophagogastric anastomosis. World J Surg. 1995;19(5):745-747.

10. Sabanathan S, Mearns AJ, Richardson J. Endoscopic oesophagectomy through a right thoracoscopic approach. $J$ R Coll Surg Edinb. 1992;37(4):284-285.

11. DePaula AL, Hashiba K, Ferreira EA, de Paula RA, Grecco E. Laparoscopic transhiatal esophagectomy with esophagogastroplasty. Surg Laparosc Endosc. 1995;5(1):1-5.

12. Zingg U, McQuinn A, DiValentino D, et al. Minimally invasive versus open esophagectomy for patients with esophageal cancer. Ann Thorac Surg. 2009;87(3):911-919.

13. Wang J, Liu T, Xing M. Video-assisted thoracic surgery in the management of chest diseases. Zhonghua Yi Xue Za Zhi. 1999;79(8):589-591. Chinese.

14. Li H, Hu B, You B, Miao JB, Fu YL, Chen QR. Combined laparoscopic and thoracoscopic Ivor Lewis esophagectomy for esophageal cancer: initial experience from the People's Republic of China. Chin Med J (Engl). 2012;125(8):1376-1380.

15. Li H, Hu B, You B, et al. Completely minimally invasive Ivor Lewis esophagectomy: the preliminary experience of circular-stapled anastomosis with the trans-oral anvil. Zhonghua Wai Ke Za Zhi. 2010;48(22):1747-1750. Chinese.

16. Du JJ, Meng L, Chen JH, et al. Hand-assisted video-thoracoscopy for resection of esophageal cancer. Zhonghua Wai Ke Za Zhi. 2005;43(6): 351-353. Chinese.

17. Liu BX, Li Y, Qin JJ, et al. Comparison of thoracolaparoscopic and open three-field subtotal esophagectomy for esophageal cancer. Zhonghua Wei Chang Wai Ke Za Zhi. 2012;15(9):938-942. Chinese.

18. Hou YL, Zhao JQ, Guo W, Zang B, Tang DR. Comparison of the shortterm outcomes of patients with esophageal cancer after subtotal esophagectomy via thoracoscopy in left lateral position and in prone position. Zhonghua Wei Chang Wai Ke Za Zhi. 2012;15(9):950-953. Chinese.

19. Xue L, Pan T, Xu Z, et al. Multi-factor investigation of early postoperative cardiac arrhythmia for elderly patients with esophageal or cardiac carcinoma. World J Surg. 2009;33(12):2615-2619.

20. Wu B, Xue L, Qiu M, et al. Video-assisted mediastinoscopic transhiatal esophagectomy combined with laparoscopy for esophageal cancer. J Cardiothorac Surg. 2010;5:132.

21. Mao T, Fang WT, Gu ZT, Yao F, Guo XF, Chen WH. Comparative study of perioperative complications and lymphadenectomy between minimally invasive esophagectomy and open procedure. Zhonghua Wei Chang Wai Ke Za Zhi. 2012;15(9):922-925. Chinese.

22. Zhou J, Chen H, Lu JJ, et al. Application of a modified McKeown procedure (thoracoscopic esophageal mobilization three-incision esophagectomy) in esophageal cancer surgery: initial experience with 30 cases. Dis Esophagus. 2009;22(8):687-693.

23. Tan LJ, Wang Q, Feng MX, et al. Video-assisted thoracoscopic esophagectomy in esophageal carcinoma. Zhonghua Wei Chang Wai Ke Za Zhi. 2008;11(1):24-27. Chinese.

24. Wang H, Feng M, Tan L, Wang Q. Comparison of the short-term quality of life in patients with esophageal cancer after subtotal esophagectomy via video-assisted thoracoscopic or open surgery. Dis Esophagus. 2010;23(5):408-414. 
25. Feng MX, Wang H, Zhang Y, Tan LJ, Xu ZL, Qun W. Minimally invasive esophagectomy for esophageal squamous cell carcinoma: a case-control study of thoracoscope versus mediastinoscope assistance. Surg Endosc. 2012;26(6):1573-1578.

26. Wang H, Tan L, Feng M, Zhang Y, Wang Q. Comparison of the shortterm health-related quality of life in patients with esophageal cancer with different routes of gastric tube reconstruction after minimally invasive esophagectomy. Qual Life Res. 2011;20(2):179-189.

27. Feng MX, Tan LJ, Wang H, et al. Comparison of thoracoscopic esophagectomy in decubitus position with prone position. Zhonghua Wei Chang Wai Ke Za Zhi. 2011;14(9):686-688. Chinese.

28. Shen Y, Zhang Y, Tan L, et al. Extensive mediastinal lymphadenectomy during minimally invasive esophagectomy: optimal results from a single center. J Gastrointest Surg. 2012;16(4):715-721.

29. Feng M, Shen Y, Wang H, et al. Thoracolaparoscopic esophagectomy: is the prone position a safe alternative to the decubitus position? J Am Coll Surg. 2012;214(5):838-844.

30. Wang H, Tan LJ, Li JP, et al. Evaluation of safety of video-assisted thoracoscopic esophagectomy for esophageal carcinoma. Zhonghua Wei Chang Wai Ke Za Zhi. 2012;15(9):926-929. Chinese.

31. Zhu CC, Chen SL, Ye MH. Esophagectomy combined with radical lymphadenectomy by video-thoracoscopy. Zhonghua Wai Ke Za Zhi. 2005;43(10):628-630. Chinese.

32. Chen BF, Zhu CC, Wang CG, et al. Clinical comparative study of minimally invasive esophagectomy versus open esophagectomy for esophageal carcinoma. Zhonghua Wai Ke Za Zhi. 2010;48(16): 1206-1209. Chinese.

33. Zhu CC, Chen BF, Kong M, et al. Neoadjuvant chemoradiotherapy followed by combined thoracoscopic and laparoscopic esophagectomy in the treatment of locally advanced esophageal carcinoma. Zhonghua Wei Chang Wai Ke Za Zhi. 2012;15(9):943-946. Chinese.

34. Lin J, Kang M, Chen C, et al. Thoracolaparoscopy oesophagectomy and extensive two-field lymphadenectomy for oesophageal cancer: Introduction and teaching of a new technique in a high-volume centre. Eur J Cardiothorac Surg. 2013;43(1):115-121.

35. Liu SY, Wang F, Zheng QF, Chen XF. Clinical application of minimally invasive esophagectomy for esophageal carcinoma. Zhonghua Wei Chang Wai Ke Za Zhi. 2012;15(9):947-949. Chinese.

36. Lin JB, Kang MQ, Lin RB, Zhen W, Chen C. Thoracolaparoscopic esophagectomy with two-field lymph node dissection for esophageal carcinoma: report of 150 cases. Zhonghua Wei Chang Wai Ke Za Zhi. 2012;15(9):930-933. Chinese.

37. Lin FC, Russell H, Ferguson MK. Strangulation of the reconstructive gastric tube by the azygos arch. Ann Thorac Surg. 2006;82(2):e8-e10.

38. Cense HA, Law S, Wei W, et al. Pharyngo-laryngo-esophagectomy using the thoracoscopic approach. Surg Endosc. 2007;21(6):879-684.
39. Law SY, Fok M, Wei WI, et al. Thoracoscopic esophageal mobilization for pharyngo-laryngo-esophagectomy. Ann Thorac Surg. 2000;70(2): 418-422.

40. Wong SK, Chan AC, Lee DW, To EW, Ng EK, Chung SC. Minimal invasive approach of gastric and esophageal mobilization in total pharyngo-laryngo-esophagectomy: total laparoscopic and hand-assisted laparoscopic technique. Surg Endosc. 2003;17(5):798-802.

41. Wang YB, Cai RJ, Han YJ, Wang WJ, Yang XY, Liu SE. Quality of life in patients with esophageal carcinoma undergoing thoracoscopic and laparoscopic esophagectomy and circular stapled cervical esophagogastric anastomosis via retrosternal route. Zhonghua Wei Chang Wai Ke Za Zhi. 2011;14(6):428-431. Chinese.

42. Xie X, Fu JH, Wang JY, et al. Analysis of learning process of videoassisted minimally invasive esophagectomy for thoracic esophageal carcinoma. Zhonghua Wei Chang Wai Ke Za Zhi. 2012;15(9):918-921. Chinese.

43. Yuan YC, Xia ZK, Yin N, Yin BL, Hu JG. Modified thoracoscopic versus minimally invasive oesophagectomy in curative resection of oesophageal cancer. J Int Med Res. 2011;39(3):904-911.

44. Guo W, Zou YB, Ma Z, et al. One surgeon's learning curve for videoassisted thoracoscopic esophagectomy for esophageal cancer with the patient in lateral position: how many cases are needed to reach competence? Surg Endosc. October 24, 2012. [Epub ahead of print.]

45. Guo W, Zhao YP, Jiang YG, et al. Prevention of postoperative chylothorax with thoracic duct ligation during video-assisted thoracoscopic esophagectomy for cancer. Surg Endosc. 2012;26(5):1332-1326.

46. Zhang ZM, Wang Y, Gao YS, Song Y, Ma L. Minimally invasive esophagectomy for esophageal carcinoma: clinical analysis of 160 cases. Zhonghua Wei Chang Wai Ke Za Zhi. 2012;15(9):934-937. Chinese.

47. Wang ZQ, Zhang YC, Zhang Q, Zhu XJ, Shu Y, Zhou ZG. Laparoscopic transhiatal extended gastrectomy for type II, III esophagogastric junction cancer: a preliminary report of 55 cases. Zhonghua Wei Chang Wai Ke Za Zhi. 2010;13(9):652-655. Chinese.

48. Gao Y, Wang Y, Chen L, Zhao Y. Comparison of open three-field and minimally-invasive esophagectomy for esophageal cancer. Interact Cardiovasc Thorac Surg. 2011;12(3):366-369.

49. Ren MY, Huang B, Zhang J, Pu M, Deng SH. Laparoscopic transhiatal proximal gastrectomy for adenocarcinoma of the esophagogastric junction: report of 98 cases. Zhonghua Wei Chang Wai Ke Za Zhi. 2012;15(9):906-909. Chinese.

50. Hulscher JB, van Sandick JW, de Boer AG, et al. Extended transthoracic resection compared with limited transhiatal resection for adenocarcinoma of the esophagus. $N$ Engl J Med. 2002;347(21):1662-1669.

51. Hulscher JB, Tijssen JG, Obertop H, van Lanschot JJ. Transthoracic versus transhiatal resection for carcinoma of the esophagus: a metaanalysis. Ann Thorac Surg. 2001;72(1):306-313.
OncoTargets and Therapy

\section{Publish your work in this journal}

OncoTargets and Therapy is an international, peer-reviewed, open access journal focusing on the pathological basis of all cancers, potential targets for therapy and treatment protocols employed to improve the management of cancer patients. The journal also focuses on the impact of management programs and new therapeutic agents and protocols on

\section{Dovepress}

patient perspectives such as quality of life, adherence and satisfaction. The manuscript management system is completely online and includes a very quick and fair peer-review system, which is all easy to use. Visit http://www.dovepress.com/testimonials.php to read real quotes from published authors. 